\title{
How Does Bony Surgery Affect Results of Anterior Open Reduction in Walking-age Children With Developmental Hip Dysplasia?
}

\author{
Alpesh Kothari MRCS, MSc, George Grammatopoulos FRCS (Tr \& Orth), DPhil, \\ Sally Hopewell DPhil, Tim Theologis MSc, PhD
}

Published online: 20 October 2015

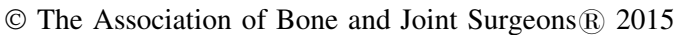

\begin{abstract}
Background Anterior open reduction is commonly used to treat hip subluxation or dislocation in developmental dysplasia of the hip (DDH) in walking-age children. Pelvic and/ or femoral osteotomy may be used in addition, but it is unclear how this affects avascular necrosis (AVN) risk and radiological and clinical results.

Questions/purposes The purpose of this study was to review studies of walking-age patients treated either with an open reduction alone or combined with pelvic and/or femoral osteotomies and determine whether there is a difference between groups in the proportion of patients: (1) developing clinically relevant femoral head AVN (Kalamchi \& MacEwen Types II to IV or equivalent); (2) achieving a satisfactory radiological result (Severin Grade I/II or equivalent); (3) achieving a satisfactory clinical result (McKay excellent or good rating or equivalent); and (4) requiring further nonsalvage surgery.

Methods MEDLINE, Embase, the Cochrane Centre Register of Controlled Trials, and ClinicalTrials.gov were searched for studies of anterior open reduction for DDH in children aged 12 months to 6 years old. We assessed AVN,
\end{abstract}

Each author certifies that he or she, or a member of his or her immediate family, has no funding or commercial associations (eg, consultancies, stock ownership, equity interest, patent/licensing arrangements, etc) that might pose a conflict of interest in connection with the submitted article.

All ICMJE Conflict of Interest Forms for authors and Clinical Orthopaedics and Related Research ${ }^{\circledR}$ editors and board members are on file with the publication and can be viewed on request.

A. Kothari ( $₫)$, G. Grammatopoulos, S. Hopewell, T. Theologis

Nuffield Orthopaedic Centre, Windmill Road,

Oxford OX3 7LD, UK

e-mail: Alpesh.kothari@ndorms.ox.ac.uk clinical and radiological results, and requirement for further procedures. The effect of failed conservative management, traction, age at operation, and followup duration was also assessed. Eighteen studies met the review eligibility criteria. Results Open reduction alone had a lower risk of AVN than open reduction combined with pelvic and femoral osteotomy ( $4 \%$ versus $24 \%$ ), but there was no significant difference compared with open reduction with either pelvic $(17 \%)$ or femoral osteotomy (18\%). More hips treated with open reduction alone had satisfactory radiological results than open reduction combined with pelvic and femoral osteotomy (97\% versus $83 \%$ ) and satisfactory clinical results than all other interventions. More hips treated with open reduction alone required further surgical management $(56 \%)$ compared with open reduction and pelvic osteotomy (11\%) and combined pelvic and femoral osteotomies (8\%).

Conclusions Open reduction with concomitant pelvic osteotomy is the most appropriate option to provide durable results with the lowest risk of AVN and best radiological and clinical results. There is no evidence that addition of a femoral osteotomy provides any additional benefit to the patient, although it may be necessary to achieve reduction.

Level of Evidence Level III, therapeutic study.

\section{Introduction}

Treating hip subluxation or dislocation resulting from developmental hip dysplasia (DDH) in a child after walking age, either because of failed conservative measures [17] or late presentation despite a program of hip screening [12, 39], presents a unique and difficult challenge for the pediatric orthopaedic surgeon. Soft tissue contracture, an inverted limbus, and capsular constriction necessitate 
formal open reduction, soft tissue release, and capsulorrhaphy. A number of open reduction methods have been described with the anterior open reduction using the SmithPeterson or iliofemoral approach [37], the most commonly used in this age group. Open reduction may be performed in isolation or combined with pelvic and/or femoral osteotomy to deal with persistent acetabular dysplasia [32], proximal femoral deformity [34], and a reduction in hip remodeling potential with increasing age [15]. Surgical intervention has the risk of iatrogenic avascular necrosis (AVN) of the femoral head, which in turn adversely affects the final clinical and radiological result [29, 44]. The use of traction or shortening at the proximal femoral osteotomy site may reduce this risk $[19,35]$.

There is limited guidance on how and in what combination these procedures are used to best effect. There are numerous advocates of a single-stage surgical approach to this problem combining open reduction and pelvic and femoral osteotomy, to minimize need for future intervention [11, 12, 39]. Others deem this too aggressive an approach and base management on intraoperative stability and subjective assessment of soft tissue tension [22, 45]. Ultimately the surgical aim is to undertake the minimum amount of intervention necessary to obtain a stable concentric reduction, avoiding AVN or further surgical procedures to provide a pain-free hip that will last decades.

The effectiveness of these different surgical interventions is varied with reports of the incidence of clinically relevant AVN (Kalamchi \& MacEwen Types II to IV or equivalent) varying from $0 \%$ to $39 \%[5,22,26]$, a satisfactory radiological result (Severin Grade I/II) $83 \%$ to $96 \%$ $[10,32]$, and a satisfactory clinical result (McKay rating excellent or good) $71 \%$ to $100 \%$ [5, 44]. Reports often pool results from a variety of different surgical approaches undertaken in ill-defined patient groups with broad age ranges and varying degrees of followup [25, 27, 35]. Given the complexities of the data reported, it is difficult to appraise the literature to appropriately advise parents on the effectiveness and potential harms associated with surgical intervention their child may face.

The aim of this systematic review was to determine in studies of walking-age patients with DDH who were treated either with an open reduction alone or an open reduction combined with femoral and/or pelvic osteotomies, whether there were differences between the groups in terms of (1) the proportion of patients developing femoral head AVN (Kalamchi \& MacEwen Types II-IV or equivalent); (2) the proportion of patients achieving a satisfactory radiological result (Severin I/II or equivalent); (3) the proportion of patients achieving a satisfactory clinical result (McKay excellent or good rating, or equivalent); and (4) the requirement for further nonsalvage surgical procedures. We also endeavored to assess the contributions of age at surgical intervention, duration of followup, traction, and failed previous conservative management on the measured endpoints.

\section{Search Strategy and Study Selection Criteria}

This systematic review is reported in accordance with PRISMA guidelines (www.prisma-statement.org/).

A comprehensive search of the literature was undertaken in MEDLINE, Embase, the Cochrane Centre Register of Controlled Trials (CENTRAL), and ClinicalTrials.gov. The MEDLINE and Embase searches were undertaken in the OvidSP search platform from the earliest date available until the second week of January 2015 (Table 1). The website-specific search engines were used for the remaining two databases. Synonyms for DDH as well as relevant subject headings were used in combination with appropriate truncations, wildcards, and Boolean operators to identify relevant articles. All published conference proceedings were included as part of the search strategy, non-English language studies were excluded, and reference lists of review articles and article

Table 1. Search strategy with article numbers for Medline and Embase searches including fields, truncations, wildcards, and Boolean operators

\begin{tabular}{|c|c|c|c|}
\hline Number & Search term & Medline & Embase \\
\hline 1 & hip\$.mp & 290,222 & 372,873 \\
\hline 2 & congenital.mp & 247,368 & 414,049 \\
\hline 3 & development\$.mp & $1,746,649$ & $2,347,493$ \\
\hline 4 & dysplas\$.mp & 70,422 & 105,431 \\
\hline 5 & dislocate\$.mp & 54,908 & 61,857 \\
\hline 6 & \$luxation.mp & 3752 & 5602 \\
\hline 7 & exp hip dislocation, congenital/ & 6744 & 5089 \\
\hline 8 & exp hip dysplasia & N/A & 4260 \\
\hline 9 & 2 OR 3 & $1,961,982$ & $2,705,040$ \\
\hline 10 & 4 OR 5 OR 6 & 122,828 & 165,625 \\
\hline 11 & $(1 \operatorname{adj} 5$ (9) $\operatorname{adj} 5(10)) \cdot m p$ & 8058 & 7735 \\
\hline 12 & 7 OR 8 OR 11 & 8058 & 10,207 \\
\hline 13 & (open adj2 reduc\$).mp & 8305 & 12,508 \\
\hline 14 & 12 and 13 & 413 & 563 \\
\hline \multirow[t]{2}{*}{15} & $\begin{array}{l}\text { limit } 14 \text { to (human and } \\
\text { child }<\text { unspecified age }>\text { ) }\end{array}$ & N/A & 216 \\
\hline & $\begin{array}{l}\text { limit } 13 \text { to ("all child [0 to } 18 \\
\text { years]" and humans) }\end{array}$ & 358 & N/A \\
\hline 16 & $\begin{array}{l}\text { (newborn } \$ \text { or neonat } \$ \text { or infan } \$ \text { or } \\
\text { teen } \$ \text { or adolescent } \$ \text { or toddler } \$ \\
\text { or tot } \$ \text { or boy } \$ \text { or girl or lad\$ or } \\
\text { youth or p?ediatric } \$ \text { ).mp }\end{array}$ & $4,017,175$ & $4,327,125$ \\
\hline 17 & 15 OR (14 AND 16) & 378 & 439 \\
\hline
\end{tabular}

$\mathrm{N} / \mathrm{A}=$ not applicable. 
meeting the inclusion criteria were screened to highlight any further relevant studies not catalogued by the search strategy.

Abstracts identified from the initial literature search were screened by two investigators (AK, GG) for potential eligibility based on the primary inclusion criteria of children with DDH having primary treatment by anterior open reduction and reporting clinical results, radiological results, and/or incidence of AVN.

For the purposes of this review, the age range selected was 12 months to 6 years old. The lower limit of 12 months was selected because this is consistent with the normal mean walking age as described by the World Health Organization [43]. The upper age limit was selected because it is believed that after this age, it is unclear whether surgical intervention for late-presenting $\mathrm{DDH}$ is preferable to nonoperative management [16, 23, 32]. Studies that included surgical intervention with nonsalvage pelvic osteotomy (Salter innominate, Pemberton, and Dega) and/or proximal femoral osteotomy were included. Studies were required to report a minimum mean followup of greater than 5 years. This was to ensure the inclusion of late-onset signs of AVN. Studies were required to report on the results of anterior open reduction of at least 10 patients. All study designs were considered for inclusion in the review.

Exclusion criteria included surgery in only teratological or neuromuscular hips, revision open reduction, open reduction, which included limbusectomy, and an alternative surgical approach to the ones specified. Review articles were identified and excluded.

If it was unclear from the abstract whether the article met either the inclusion or exclusion criteria, then the full article was retrieved. If the abstract reported results from multiple surgical techniques, including open reduction, or had an age range that went lower than 12 months or older than 6 years old, then again the full article was retrieved to assess whether groups of patients or raw individual patient data could be identified that did fulfill the inclusion criteria.

The full-text articles were then reviewed by the same two investigators (AK, GG) to ensure inclusion criteria could be met by all or part of the data presented. Quality assessment of the shortlisted articles was performed independently using critical appraisal tools from the Centre of Evidence Based Medicine (CEBM) (www.cebm.net/ critical-appraisal). To be included in the review, the article had to fulfill more than half of the criteria outlined by the assessment tool. Disagreements at any stage of the article selection process were resolved by the senior author (TT). Data extraction was undertaken by one of the authors (AK) using a standardized pro-forma, which had been piloted by two of the authors (AK, GG) before use. Extracted data were entered into an MS Excel spreadsheet (Microsoft Inc, Redmond, WA, USA).
The literature search yielded 824 citations of potential studies (439 from Embase, 378 from MEDLINE, seven from Central) (Fig. 1). Searching ClinicalTrials.gov yielded one potentially relevant trial, although there were no recorded data or publication associated with the database entry. After assessment of the title and abstract, 695 recorded were excluded as a result of duplication, article only available in a foreign language, inadequate followup, alternative intervention, alternative age group, or case report (Fig. 1). Twenty-five review articles were identified, and from the reference lists of these articles, another three eligible studies were identified. After full-text review, a further 72 articles were excluded leaving 18 studies that met all the inclusion criteria and quality assessment using the CEBM critical appraisal tool (Table 2) [3, 5, 7, 10, 11, 14, 22, 26, 28, 30-32, 38-40, 44-46]. All the studies identified were retrospective in design. Using the criteria outlined by Dekkers et al. [9], 17 studies could be broadly classified as cohort studies and one as a case series [46]. Nine of these studies were comparative, investigating the differences among different surgical indications, interventions, or patient populations $[3,11,22,26,28,31,38,40,46]$. For six of these studies, only selected subgroup data fulfilled the inclusion criteria [3, 26, 28, 31, 40, 46]. In total, data were available on 1048 hips in over 806 patients who had an anterior open reduction of the hip for $\mathrm{DDH}$. Individual patient data were available for 149 patients from seven studies [3, 28, 30, 31, 39, 40, 46].

The 18 studies included reported the presence of AVN using a variety of methods including the Kalamchi and MacEwen (K\&M) [18], Bucholz and Ogden (B\&O) [6], and Salter classifications [33] (Table 3). For the purposes of this review, AVN was recorded as clinically relevant if it was classified as $\mathrm{K} \& \mathrm{M}$ or B\&O Grade II or greater. The studies that used the Salter classification did not report the specific pattern of AVN; thus, it was assumed that all the cases represented clinically relevant AVN. Barrett et al. [3] used a binary classification for AVN, and again if present, it was assumed to be clinically relevant. Zionts et al. [46] described AVN in terms of either temporary irregular ossification (TIO) or central AVN. Central AVN was deemed clinically relevant, whereas TIO was deemed to be equivalent to a K\&M Type I.

The radiological appearance of the hip was recorded in all but one study [38] (Table 3). The majority of studies used the Severin score to measure radiological result [36]. In accordance with a number of previous authors, Severin Class I and II were considered as satisfactory and Grade III to VI as unsatisfactory radiological result [2, 13, 20]. Subasi et al. [39] reported the radiological appearance of the hip using the center-edge angle (CEA) and arch index. A CEA of $>20^{\circ}$ was recorded as Severin II or better 


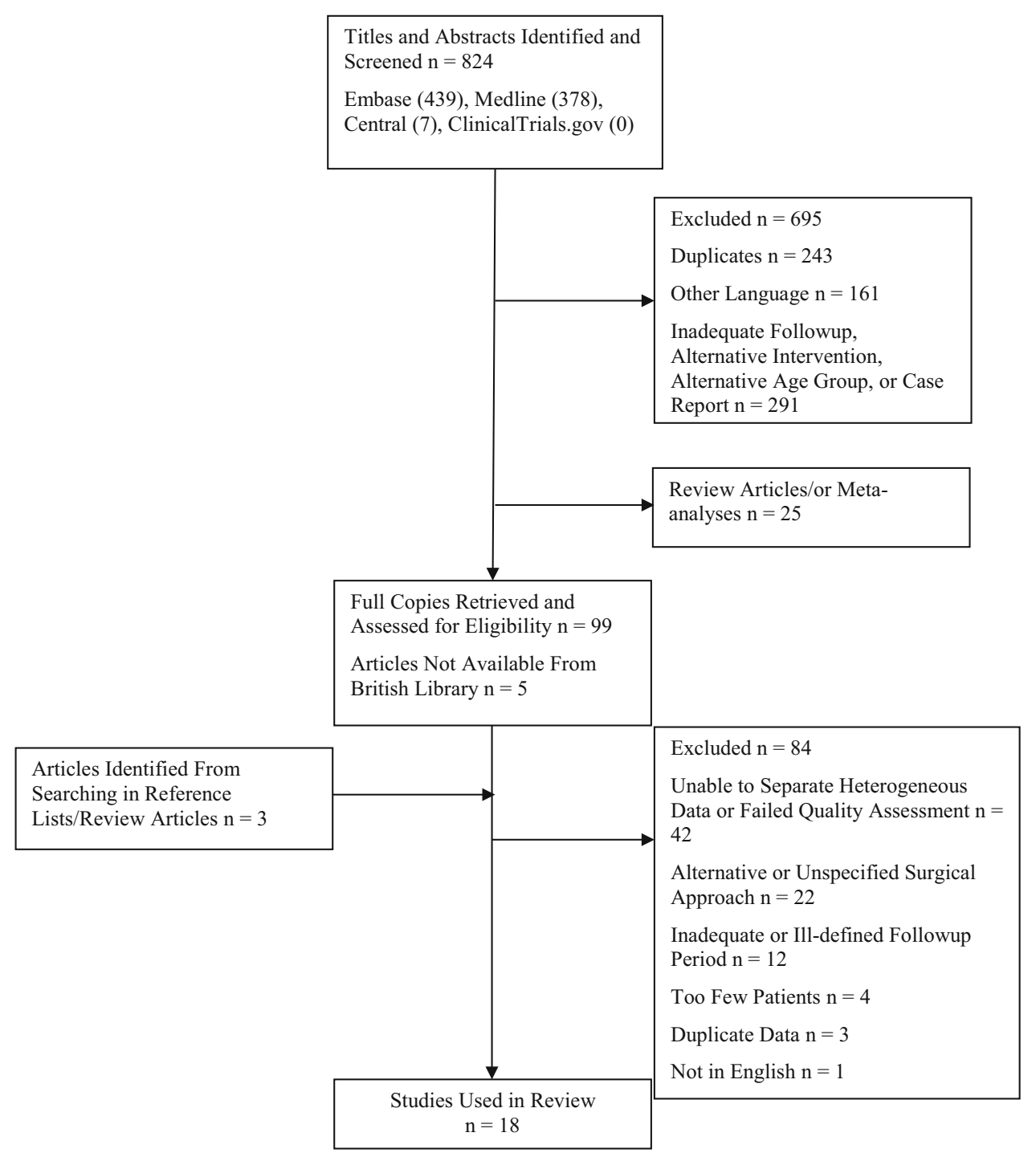

Fig. 1 A flowchart demonstrating the systematic review search strategy and depicting results of the literature search and study selection process.

(satisfactory radiological result). Powell et al. [28] recorded radiological appearance as excellent, fair, or poor on the basis of any residual subluxation $\left(\mathrm{CEA}<20^{\circ}\right)$, acetabular dysplasia (acetabular index of $\leq 25^{\circ}$ ), and evidence of AVN. For the purposes of this review, hips defined as excellent or fair were considered as a satisfactory radiological result.

Clinical results were recorded in 15 of the 18 studies used in the review (Table 3). Thirteen of these used the McKay score [23]. These scores were also dichotomized in accordance with Erturk et al. [11] with an excellent or good rating being regarded as a satisfactory clinical result and fair or poor ratings as clinically unsatisfactory. Ryan et al. [31] measured clinical outcome using the Iowa Hip Score (IHS) [21]. Patients with an IHS between 80 and 100 were rated as a satisfactory clinical result (90 to 100 [excellent], 80 to 89 [good]), and those with a score below 80 as an unsatisfactory clinical result. Subasi et al. [39] used the Trevor score [41] to measure the results of surgery, but because this is a composite clinical and radiological score, it could not be used to quantify solely clinical result for the purposes of this review.

The overall percentage of clinically relevant AVN, proportion of satisfactory clinical and radiological results, and requirement for further nonsalvage surgical intervention were assessed for all procedures involving open reduction for DDH. Data from the studies were then pooled according to the specific surgery, open reduction alone, open reduction and pelvic osteotomy, open reduction and proximal femoral osteotomy, or open reduction and both femoral and pelvic osteotomy. As a result of the nature of data available, formal meta-analysis was not possible. However, to put the review findings in context, some statistical analysis was undertaken. Multiple Fisher's exact 
Table 2. Summary of quality assessment for studies included in systematic review based on Centre of Evidence Based Medicine case study appraisal tool (www.cebm.net/critical-appraisal)*

Appraisal question
Did the study address a clearly focused question/issue?
Is the research method (study design) appropriate for answering the
research question?
Are both the setting and the subjects representative with regard to the
population to which the findings will be referred?
Ar the researcher's perspective clearly described and taken into account?
Are the methods for collecting data clearly described?
quality control measures used?
Was the analysis repeated by more than one research to ensure reliability?
Are the conclusions justified by the results?
Are the findings of the study transferable to other settings?

* Study corresponding to study number as follows: (1) Barrett et al. [3]; (2) Berkeley et al. [5]; (3) Chang et al. [7]; (4) El-Sayed [10]; (5) Erturk et al. [11]; (6) Haidar et al. [14]; (7) Lin et al. [22]; (8) Ning et al. [26]; (9) Powell et al. [28]; (10) Ruszkowski and Pucher [30]; (11) Ryan et al. [31]; (12) Salter and Dubos [32]; (13) Spence et al. [38]; (14) Subasi et al. [39]; (15) Tezeren et al. [40]; (16) Wu et al. [44]; (17) Zadeh et al. [45]; (18) Zionts et al. [46].

tests were used to investigate the relationship between surgical intervention and proportion of hips with the endpoint of interest. Odds ratios (ORs) with 95\% confidence intervals (CIs) were also calculated.

Where raw individual patient data were available, univariate analysis was undertaken using either the Student's t-test or Fisher's exact test to assess the relationship among age at operation, duration of followup, need for further procedures, preoperative traction, and failed previous closed reduction on AVN rates, clinical results, and radiological results. Continuous data were assessed for normality, kurtosis using standard testing procedures. Multivariate analysis was not possible as a result of the small sample of individual patient data. Fisher's exact test was used to assess the relationship between AVN and clinical and radiological outcome. False discovery rate was controlled for by using the Benjamini and Yekutieli method [4] such that the corrected overall critical $p$ value was 0.003 . Results below this level were regarded as statistically significant. Statistical analyses were undertaken using STATA Version 13.0 (Statacorp LP, College Station, TX, USA).

\section{Results}

Open reduction alone was associated with a lower risk of clinically relevant AVN (K\&M Type II-IV or equivalent) (4\% [three of 69 hips]) than open reduction with both pelvic and femoral osteotomies (24\% [86 of 366 hips]) (OR, 0.15; 95\% CI, 0.05-0.48; p < 0.001) (Tables 4, 5).
There was no difference in the risk of AVN after open reduction alone (4\% [three of 69 hips]) compared with open reduction with pelvic osteotomy (17\% [50 of 290 hips]) (OR, 0.21; 95\% CI, 0.07-0.72; p = 0.004) or compared with open reduction with femoral osteotomy $(18 \%$ [12 of 67 hips]) (OR, 0.21; 95\% CI, 0.15-20.51; p = 0.014). The overall risk of clinically relevant AVN for open reduction in DDH for children from 12 months to 6 years old, inclusive of all combinations of pelvic and femoral osteotomy, was $17 \%$ (157 of 938 hips).

Open reduction alone was also associated with the increased odds of a satisfactory radiological result (Severin I or II or equivalent) (97\% [67 of 69 hips]) compared with open reduction with both pelvic and femoral osteotomies (83\% [304 of 366 hips]) (OR, 6.83; 95\% CI, 1.63-28.63; $\mathrm{p}=0.001)$ (Tables 4,5$)$. There was no difference in the odds of a satisfactory radiological result after open reduction alone (97\% [67 of 69 hips]) compared with open reduction with pelvic osteotomy (88\% [321 of 363 hips]) (OR 4.38; 95\% CI, 1.04-18.55; $\mathrm{p}=0.029$ ) or open reduction with femoral osteotomy (95\% [19 of 20 hips]) (OR 1.76; 95\% CI, 0.15-20.51; p = 0.539). The overall percentage of hips with a satisfactory radiological result was $86 \%$ (825 of 964 hips).

Open reduction alone was associated with increased odds of a satisfactory clinical result (McKay score excellent or fair or equivalent) (100\% [58 of 58 hips]) compared with open reduction with pelvic osteotomy $(86 \%$ [205 of 238 hips]) (OR, 19.07; 95\% CI, 1.15-316.02; $\mathrm{p}=0.001$ ), open reduction with femoral osteotomy (50\% [two of four hips]) (OR 117.00; 95\% CI, 4.35-3139.68; p = 0.003), and 


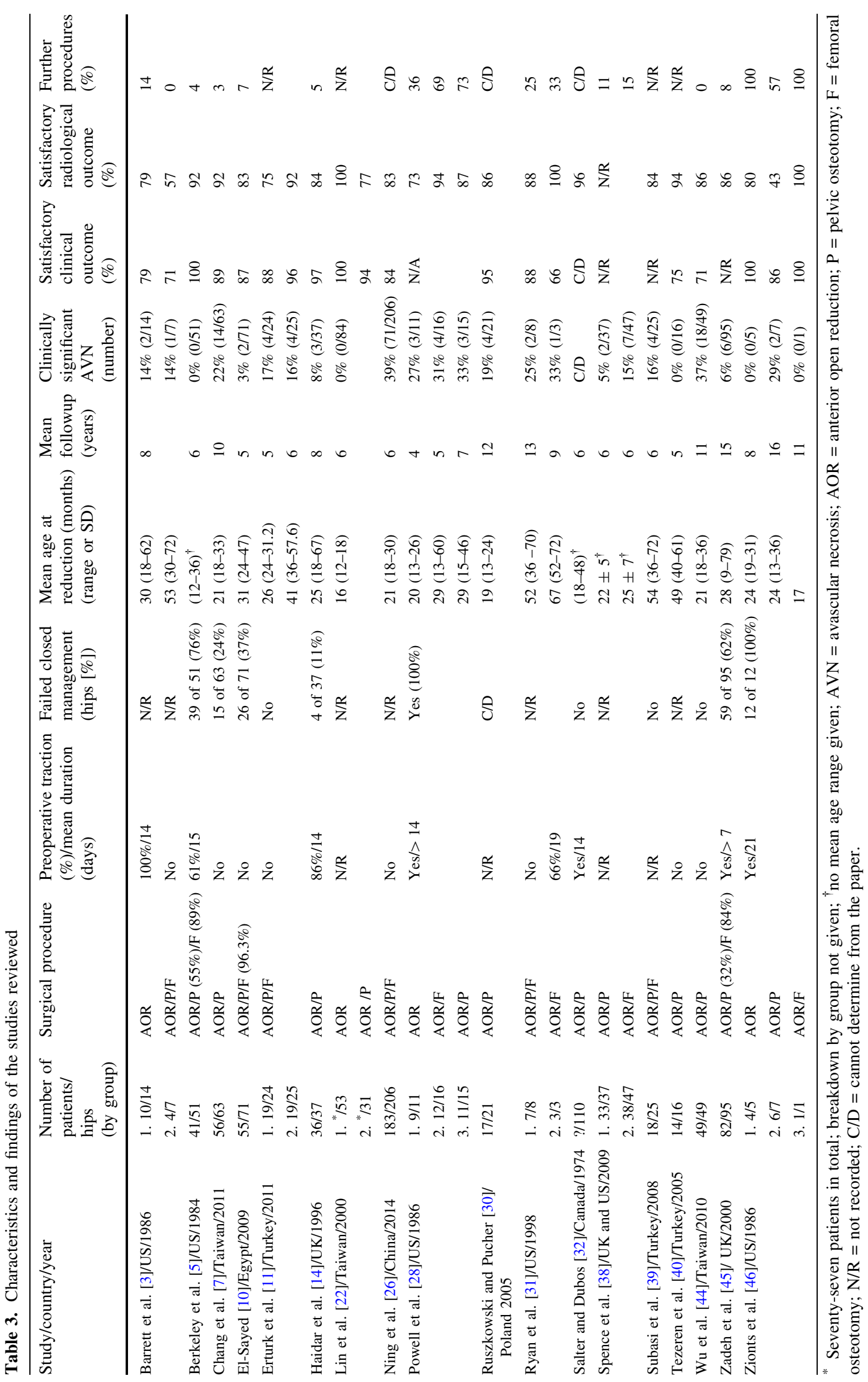


Table 4. Summary table demonstrating AVN rates, radiological and clinical results, and rate/further procedures by type/surgery*

\begin{tabular}{lllll}
\hline Parameter & \multicolumn{2}{l}{ Surgical intervention } \\
\cline { 2 - 5 } & Open reduction & $\begin{array}{l}\text { Open reduction and } \\
\text { pelvic osteotomy }\end{array}$ & $\begin{array}{l}\text { Open reduction and } \\
\text { femoral osteotomy }\end{array}$ & $\begin{array}{l}\text { Open reduction and both pelvic } \\
\text { and femoral osteotomies }\end{array}$ \\
\hline Mean age at the time/surgery (months) & 17 & 23 & 28 & 28 \\
Clinically relevant AVN (\%) & $4 \%(3 / 69)$ & $17 \%(50 / 290)$ & $18 \%(12 / 67)$ & $24 \%(86 / 366)$ \\
Satisfactory radiological result (\%) & $97 \%(67 / 69)$ & $88 \%(321 / 363)$ & $95 \%(19 / 20)$ & $83 \%(304 / 366)$ \\
Satisfactory clinical result (\%) & $100 \%(58 / 58)$ & $86 \%(205 / 238)$ & $50 \%(2 / 4)$ & $85 \%(293 / 341)$ \\
Further procedures & $56 \%(9 / 16)$ & $11 \%(25 / 222)$ & $29 \%(20 / 67)$ & $8 \%(7 / 86)$ \\
\hline
\end{tabular}

* Numbers in parentheses indicate number/hips that have outcome as numerator and total number/hips available for analysis as denominator; $\mathrm{AVN}=$ avascular necrosis.

Table 5. Results summary of comparison between surgical prescription*

\begin{tabular}{|c|c|c|c|c|c|c|}
\hline Parameter & $\begin{array}{l}\text { AOR } \\
\text { versus } \\
\text { AOR/P }\end{array}$ & $\begin{array}{l}\text { AOR } \\
\text { versus } \\
\text { AOR/F }\end{array}$ & $\begin{array}{l}\text { AOR } \\
\text { versus } \\
\text { AOR/F/P }\end{array}$ & $\begin{array}{l}\mathrm{AOR} / \mathrm{P} \\
\text { versus } \\
\mathrm{AOR} / \mathrm{F}\end{array}$ & $\begin{array}{l}\mathrm{AOR} / \mathrm{P} \\
\text { versus } \\
\mathrm{AOR} / \mathrm{F} / \mathrm{P}\end{array}$ & $\begin{array}{l}\mathrm{AOR} / \mathrm{F} \\
\text { versus } \\
\mathrm{AOR} / \mathrm{F} / \mathrm{P}\end{array}$ \\
\hline $\mathrm{AVN}$ & $0.21(0.07-0.72)$ & $0.21(0.06-0.78)$ & $0.15(0.045-0.48)$ & $0.95(0.48-1.91)$ & $0.68(0.46-1.00)$ & $0.71(0.36-1.39)$ \\
\hline & $\mathrm{p}=0.004$ & $\mathrm{p}=0.014$ & $\mathrm{p}<0.001^{\dagger}$ & $\mathrm{p}=0.856$ & $\mathrm{p}=0.052$ & $\mathrm{p}=0.345$ \\
\hline Radiological outcome & $\begin{array}{l}4.38(1.04-18.55) \\
p=0.029\end{array}$ & $\begin{array}{l}1.76(0.15-20.51) \\
p=0.539\end{array}$ & $\begin{array}{l}6.83(1.63-28.63) \\
p=0.001^{\dagger}\end{array}$ & $\begin{array}{l}0.40(0.05-3.08) \\
p=0.713\end{array}$ & $\begin{array}{l}1.56(1.02-2.38) \\
p=0.044\end{array}$ & $\begin{array}{l}3.88(0.51-29.5) \\
p=0.220\end{array}$ \\
\hline Clinical outcome & $\begin{array}{l}19.07(1.15-316.02) \\
\mathrm{p}=0.001^{\dagger}\end{array}$ & $\begin{array}{l}117.00(4.35-3139.68) \\
\mathrm{p}=0.003^{\dagger}\end{array}$ & $\begin{array}{l}19.33(1.18-318.01) \\
\mathrm{p}=0.001^{\dagger}\end{array}$ & $\begin{array}{l}6.21(0.85-45.64) \\
p=0.101\end{array}$ & $\begin{array}{l}1.02(0.63-1.64) \\
\mathrm{p}=1.000\end{array}$ & $\begin{array}{l}0.16(0.02-1.19) \\
\mathrm{p}=0.102\end{array}$ \\
\hline Further procedures & $\begin{array}{l}10.13(3.47-29.59) \\
\mathrm{p}<0.001^{\dagger}\end{array}$ & $\begin{array}{l}3.02(0.99-9.24) \\
p=0.078\end{array}$ & $\begin{array}{l}14.51(4.14-50.86) \\
\mathrm{p}<0.001^{\dagger}\end{array}$ & $\begin{array}{l}0.30(0.15-0.58) \\
\mathrm{p}<0.001^{\dagger}\end{array}$ & $\begin{array}{l}1.43(0.60-3.45) \\
\mathrm{p}=0.415\end{array}$ & $\begin{array}{l}4.80(1.89-12.21) \\
\mathrm{p}<0.001^{\dagger}\end{array}$ \\
\hline
\end{tabular}

* Level of significance adjusted to $\alpha<0.003$; ${ }^{\dagger}$ statistically significant result; odds ratio with $95 \%$ confidence intervals in parentheses; AOR = anterior open reduction; $\mathrm{P}=$ pelvic osteotomy; $\mathrm{F}=$ femoral osteotomy; $\mathrm{AVN}=$ avascular necrosis.

open reduction with both pelvic and femoral osteotomies (85\% [293 of 341 hips]) (OR 19.33; 95\% CI, 1.18-318.01; $\mathrm{p}=0.001$ ) (Tables 4,5$)$. The overall percentage of hips with a satisfactory clinical result was $87 \%$ (610 of 702 hips).

The requirement for further procedures (revision OR and/ or pelvic and/or femoral osteotomies) was higher in the open reduction alone (56\% [nine of $16 \mathrm{hips}$ ]) and open reduction with femoral osteotomy (29\% [20 of 67 hips]) groups compared with both of the other groups (Table 4). There was no difference in the requirement for further procedures in the open reduction and pelvic osteotomy group (11\% [25 of 222 hips]) compared with the open reduction with both pelvic and femoral osteotomies (8\% [seven of 86 hips]) (OR 1.43; 95\% CI, 0.60-3.45; $\mathrm{p}=0.415$ ).

Analysis of individual patient data demonstrated that failed previous conservative treatment, preoperative traction, nor the need for further procedures affected the risk of AVN or adverse clinical or radiological results. There was also no evidence that duration of followup biased risk of AVN or radiological or clinical results. The only positive finding was that children with satisfactory clinical results were younger than those with unsatisfactory results $(37.1 \pm$
17.4 months versus $51.8 \pm 16.4$ months, $\mathrm{p}=0.001)$. The presence of AVN was associated with increased odds of unsatisfactory radiological results (OR, 34.76; 95\% CI, 10.91-110.70; $\mathrm{p}<0.001)$. A satisfactory radiological result was associated with increased odds of a satisfactory clinical result (OR, 5.67; 95\% CI, 1.84-17.45; p = 0.003).

\section{Discussion}

It is generally accepted that formal open reduction of the hip is the most appropriate intervention to treat DDH in children between the ages of 12 months and 6 years old with anterior open reduction being the preferred approach. In the literature anterior open reduction has been used in isolation in this age group but has also been combined with pelvic osteotomy $[7,14,22,28,30,32,40,44,46]$, proximal femoral osteotomy [28, 31, 38, 46], or both in a single stage [3, 10, 11, 26, 31, 39]. It is unclear which surgical procedure or combination of procedures is least likely to result in clinically relevant AVN (K\&M Types IIIV or equivalent) and most likely to provide satisfactory radiological and clinical results (Severin I or II or 
equivalent and McKay excellent or good rating or equivalent, respectively), while minimizing the requirement for further surgical intervention.

The main limitation of the review was that the literature identified by our search strategy was comprised of retrospective studies of mixed quality with inherent bias. Of note the mean age of the anterior open reduction alone group was younger than the other groups, and this may have represented a bias in preference of surgical approach by the surgeon. It is accepted that an age range of 12 months to 6 years is broad and it would have been desirable to further stratify this group by age; however, the data did not allow this. It was not possible to ascertain AVN rates, clinical and radiological results, and need for further operative intervention for all 18 articles, and where available, heterogeneity was noted in the reporting of results. Data were often pooled in mixed groups, which was adequate to quote the overall outcome rates for all surgery involving an anterior open reduction [5, 26, 30, 32, 45]. However, for subgroup analysis by specific surgical prescription, additional data were excluded, and fewer studies still had individual patient data for further analysis $[3,28$, $30,31,39,40,46]$. The nature of the data precluded formal meta-analysis; nonetheless, statistical analysis was undertaken to put the findings in some context. Confidence in the review findings may also be compromised by publication bias, because the study selection criteria were likely to favor larger centers with greater amounts of experience with DDH in walking-age children. As such the results presented in this review are likely to represent the best possible scenario with the true risk of AVN and unsatisfactory radiological and clinical results potentially being worse.

It is also important to consider the multifactorial nature of AVN in this population. Other factors of potential importance, including failed conservative management, preoperative traction, and further procedures, were assessed and were not associated with outcome. However, the data set was small in size with the consequence of an increased likelihood of a Type II error. It is also accepted that the height/Tönnis grade of the dislocation, and postoperative immobilization protocols may affect results, but there was inadequate data on these variables in the articles used in this review. The technical aspects of the open reduction, including details on how the capsule and other soft tissue structures were dealt with, are also of importance, but the data were not of sufficient quality to evaluate this. Indeed, there are so many variables that could affect results that Morcuende et al [24] have argued that it is difficult to know the contribution of surgery alone to the risk of AVN. The inclusion criterion of minimum mean followup of 5 years may be regarded as inadequate to fully assess the true incidence of AVN, which may not manifest up until age 10 years [20]. This however was a pragmatic approach to the review, and duration of followup was not found to bias the risk of AVN.

Avascular necrosis of the hip is a potentially catastrophic treatment complication in DDH. It has been shown that the more profound the AVN, the worse the radiological and clinical results after intervention [7, 8, 44]. Hence, establishing which surgical prescription resulted in the lowest rates of AVN was of primary importance in this review. Open reduction alone had the lowest risk of clinically relevant AVN (K\&M Types II-IV or equivalent) in absolute terms (4\% [three of 69 hips]), but statistical analysis did not demonstrate this to be different from risk of AVN after open reduction with either pelvic or femoral osteotomy. However, open reduction with concomitant single-stage pelvic and femoral osteotomy did result in higher rates or AVN compared with open reduction alone (24\% [86 of 366 hips]). Shortening femoral osteotomy is often included to reduce the likelihood of AVN developing, but there was no evidence in this review to support this practice. The perceived benefit of mitigating any increase of pressure on the femoral head may be outweighed by the added vascular insult of disrupting metaphyseal vessels, which can persist up to the age of 4 years [42]. However, femoral shortening may be increasingly necessary with age to decrease soft tissue tension and allow stable reduction.

The importance of the final radiological appearance of the hip has been demonstrated by Albinana et al. [1] who demonstrated that a Severin classification of III or IV at skeletal maturity was over twice as likely to require THA than a Severin I or II hip. Hence, the second aim of the review was to identify the surgical procedure or combination of procedures that was associated with the highest proportion of satisfactory radiological results (Severin I or II or equivalent hips). Open reduction alone resulted in the highest proportion of satisfactory radiological results (97\% [three of 69 hips]) with open reduction and both pelvic and femoral osteotomy resulting in the lowest proportion of satisfactory radiological results (83\% [304 of 366]). These findings are in keeping with the AVN results presented in the previous paragraph and echo the findings of a number of authors $[7,8$, 44]. Indeed in this review, clinically relevant AVN was associated with a 34 times increased risk of a Severin Grade III or worse radiographic hip appearance.

From the patient and parent perspective, the clinical result of surgery is arguably more important than the radiological. Although the aim of surgery is to provide a stable pain-free hip that functions well, this is not always the result and the risk of a less than satisfactory outcome should be discussed with the patient or parent before surgery is undertaken. Thus, the third aim of this review was to ascertain which surgical prescription resulted in the highest proportion of satisfactory clinical results (McKay 
excellent or good rating or equivalent). Again anterior open reduction alone led to the best results with $100 \%$ of hips evaluated (58 of 58) having a satisfactory clinical outcome. This proved better than any other surgical prescription.

Although risk of AVN and clinical and radiological results are of utmost importance to walking-age children with DDH, it is imperative that the results of the surgery are durable. Hence, the final aim of the review was to assess the requirement for further procedures after index intervention. Over half the hips having open reduction required further nonsalvage surgical intervention. Indeed, hips undergoing open reduction alone were 10 times more likely to require further procedures than hips treated with open reduction and pelvic osteotomy and 15 times more likely than hips treated with both pelvic and femoral osteotomy. Because the inclusion criteria for mean followup duration was based on the timing of the index procedure, there may have not been adequate followup to assess the true extent of adverse sequelae from these further procedures. All measured endpoints are likely to be affected by this.

The surgical treatment of DDH in children of walking age with anterior open reduction is routinely undertaken, yet there continues to be a dearth of literature assessing how the addition of pelvic and/or femoral osteotomy affects the results. In this review, the existing literature has been evaluated in the hope that this question can be answered. From the available evidence it appears that the addition of a femoral osteotomy to the surgical plan, although potentially necessary to allow a stable reduction, may be at the detriment of increased risk of AVN (if combined with pelvic osteotomy) or further procedures (if used in isolation). Anterior open reduction alone on first appearance may seem to be the procedure of choice, but the need for further procedures is also unacceptably high. The findings of this review show that for a durable procedure with the lowest rate of AVN, and best clinical and radiological results, combining a pelvic osteotomy with anterior open reduction is the most appropriate option. This suggestion is subject to caveats and should be confirmed through well-designed prospective comparative studies.

Acknowledgments We thank Sally Ryan and Bridget Lucas for their assistance with the search strategy and retrieval of journal articles.

\section{References}

1. Albinana J, Dolan LA, Spratt KF, Morcuende J, Meyer MD, Weinstein SL. Acetabular dysplasia after treatment for developmental dysplasia of the hip. Implications for secondary procedures. J Bone Joint Surg Br. 2004;86:876-886.

2. Bache CE, Graham HK, Dickens DRV, Donnan L, Johnson MB, Nattrass G, O'Sullivan M, Torode IP. Ligamentum teres tenodesis in medial approach open reduction for developmental dislocation of the hip. J Pediatr Orthop. 2008;28:607-613.
3. Barrett WP, Staheli LT, Chew DE. The effectiveness of the Salter innominate osteotomy in the treatment of congenital dislocation of the hip. J Bone Joint Surg Am. 1986;68:79-87.

4. Benjamini Y, Yekutieli D. The control of the false discovery rate in multiple testing under dependency. Ann Stat. 2001:1165-1188.

5. Berkeley ME, Dickson JH, Cain TE, Donovan MM. Surgical therapy for congenital dislocation of the hip in patients who are twelve to thirty-six months old. J Bone Joint Surg Am. 1984;66:412-420.

6. Bucholz R, Ogden J. Patterns of ischemic necrosis of the proximal femur in nonoperatively treated congenital hip disease. In: The Hip. Proceedings of the Sixth Open Scientific Meeting of the Hip Society. St Louis, MO, USA: CV Mosby; 1978:43-63.

7. Chang $\mathrm{CH}$, Kao HK, Yang WE, Shih $\mathrm{CH}$. Surgical results and complications of developmental dysplasia of the hip-one stage open reduction and Salter's osteotomy for patients between 1 and 3 years old. Chang Gung Med J. 2011;34:84-92.

8. Cooperman DR, Wallensten R, Stulberg SD. Post-reduction avascular necrosis in congenital dislocation of the hip. $J$ Bone Joint Surg Am. 1980;62:247-258.

9. Dekkers OM, Egger M, Altman DG, Vandenbroucke JP. Distinguishing case series from cohort studies. Ann Intern Med. 2012;156:37-40.

10. El-Sayed MM. Single-stage open reduction, Salter innominate osteotomy, and proximal femoral osteotomy for the management of developmental dysplasia of the hip in children between the ages of 2 and 4 years. J Pediatr Orthop B. 2009;18: $188-196$

11. Erturk C, Altay MA, Yarimpapuc R, Koruk I, Isikan UE. Onestage treatment of developmental dysplasia of the hip in untreated children from two to five years old. A comparative study. Acta Orthop Bel. 2011;77:464-471.

12. Forlin E, Munhoz da Cunha LA, Figueiredo DC. Treatment of developmental dysplasia of the hip after walking age with open reduction, femoral shortening, and acetabular osteotomy. Orthop Clin North Am. 2006;37:149-160.

13. Gardner RO, Bradley CS, Howard A, Narayanan UG, Wedge JH, Kelley SP. The incidence of avascular necrosis and the radiographic outcome following medial open reduction in children with developmental dysplasia of the hip: a systematic review. Bone Joint J. 2014;96:279-286.

14. Haidar RK, Jones RS, Vergroesen DA, Evans GA. Simultaneous open reduction and Salter innominate osteotomy for developmental dysplasia of the hip. J Bone Joint Surg Br. 1996;78:471-476.

15. Harris NH. Acetabular growth potential in congenital dislocation of the hip and some factors upon which it may depend. Clin Orthop Relat Res. 1976;119:99-106.

16. Herold HZ, Daniel D. Surgical correction of congenital dislocation of the hip in children aged two to six years. Isr J Med Sci. 1977;13:283-289.

17. Herring JA. Tachdjian's Pediatric Orthopaedics: From the Texas Scottish Rite Hospital for Children. Philadelphia, PA, USA: Elsevier Health Sciences; 2013.

18. Kalamchi A, MacEwen GD. Avascular necrosis following treatment of congenital dislocation of the hip. J Bone Joint Surg Am. 1980;62:876-888.

19. Kaneko H, Kitoh H, Mishima K, Matsushita M, Ishiguro N. Long-term outcome of gradual reduction using overhead traction for developmental dysplasia of the hip over 6 months of age. $J$ Pediatr Orthop. 2013;33:628-634.

20. Koizumi W, Moriya H, Tsuchiya K, Takeuchi T, Kamegaya M, Akita T. Ludloff's medial approach for open reduction of congenital dislocation of the hip. A 20-year follow-up. J Bone Joint Surg Br. 1996;78:924-929.

21. Larson CB. Rating scale for hip disabilities. Clin Orthop Relat Res. 1963;31:85-96. 
22. Lin CJ, Lin YT, Lai KA. Intraoperative instability for developmental dysplasia of the hip in children 12 to 18 months of age as a guide to Salter osteotomy. J Pediatr Orthop. 2000;20:575-578.

23. McKay DW. A comparison of the innominate and the pericapsular osteotomy in the treatment of congenital dislocation of the hip. Clin Orthop Relat Res. 1974;98:124-132.

24. Morcuende JA, Meyer MD, Dolan LA, Weinstein SL. Long-term outcome after open reduction through an anteromedial approach for congenital dislocation of the hip. J Bone Joint Surg Am. 1997;79:810-817.

25. Morin C, Bisogno J, Kulkarni S, Morel G. Treatment of latepresenting developmental dislocation of the hip by progressive orthopaedic reduction and innominate osteotomy. Our results with more than 30 years of follow up. $J$ Child Orthop. 2011;5:251-260.

26. Ning B, Yuan Y, Yao J, Zhang S, Sun J. Analyses of outcomes of one-stage operation for treatment of late-diagnosed developmental dislocation of the hip: 864 hips followed for 3.2 to 8.9 years. BMC Musculoskelet Disord. 2014;15:401.

27. Pospischill R, Weninger J, Ganger R, Altenhuber J, Grill F. Does open reduction of the developmental dislocated hip increase the risk of osteonecrosis? Clin Orthop Relat Res. 2012;470:250-260.

28. Powell EN, Gerratana FJ, Gage JR. Open reduction for congenital hip dislocation: The risk of avascular necrosis with three different approaches. J Pediatr Orthop. 1986;6:127-132.

29. Ridout D, Protopapa E, Nicolaou N, Gelfer Y. Osteonecrosis complicating developmental dysplasia of the hip compromises subsequent acetabular remodeling. Clin Orthop Relat Res. 2013;471:2318-2326.

30. Ruszkowski K, Pucher A. Simultaneous open reduction and Dega transiliac osteotomy for developmental dislocation of the hip in children under 24 months of age. J Pediatr Orthop. 2005;25:695-701.

31. Ryan MG, Johnson LO, Quanbeck DS, Minkowitz B. One-stage treatment of congenital dislocation of the hip in children three to ten years old. Functional and radiographic results. J Bone Joint Surg Am. 1998;80:336-344.

32. Salter RB, Dubos JP. The first fifteen year's personal experience with innominate osteotomy in the treatment of congenital dislocation and subluxation of the hip. Clin Orthop Relat Res. 1974;98: 72-103.

33. Salter RB, Kostuik J, Dallas S. Avascular necrosis of the femoral head as a complication of treatment for congenital dislocation of the hip in young children: a clinical and experimental investigation. Can J Surg. 1969;12:44-61.

34. Sankar WN, Neubuerger CO, Moseley CF. Femoral anteversion in developmental dysplasia of the hip. $J$ Pediatr Orthop. 2009;29:885-888.

35. Schoenecker PL, Strecker W. Congenital dislocation of the hip in children. Comparison of the effects of femoral shortening and of skeletal traction in treatment. J Bone Joint Surg Am. 1984;66:21-27.

36. Severin E. Congenital dislocation of the hip. J Bone Joint Surg Am. 1950;32:507-518.

37. Smith-Petersen M. A new supra-articular subperiosteal approach to the hip joint. Am J Orthop Surg. 1917;2:592-595.

38. Spence G, Hocking R, Wedge JH, Roposch A. Effect of innominate and femoral varus derotation osteotomy on acetabular development in developmental dysplasia of the hip. J Bone Joint Surg Am. 2009;91:2622-2636.

39. Subasi M, Arslan H, Cebesoy O, Buyukbebeci O, Kapukaya A. Outcome in unilateral or bilateral DDH treated with one-stage combined procedure. Clin Orthop Relat Res. 2008;466:830-836.

40. Tezeren G, Tukenmez M, Bulut O, Percin S, Cekin T. The surgical treatment of developmental dislocation of the hip in older children: a comparative study. Acta Orthop Belg. 2005;71:678-685.

41. Trevor D, Johns D, Fixsen J. Acetabuloplasty in the treatment of congenital dislocation of the hip. J Bone Joint Surg Br. 1975;57:167-174.

42. Trueta J. The normal vascular anatomy of the human femoral head during growth. J Bone Joint Surg Br. 1957;39:358-394.

43. World Health Organisation. WHO Motor Development Study: windows of achievement for six gross motor development milestones. Acta Paediatr Suppl. 2006;450:86-95.

44. Wu KW, Wang TM, Huang SC, Kuo KN, Chen CW. Analysis of osteonecrosis following pemberton acetabuloplasty in developmental dysplasia of the hip: long-term results. J Bone Joint Surg Am. 2010;92:2083-2094.

45. Zadeh HG, Catterall A, Hashemi-Nejad A, Perry RE. Test of stability as an aid to decide the need for osteotomy in association with open reduction in developmental dysplasia of the hip. A long term review. J Bone Joint Surg Br. 2000;82:17-27.

46. Zionts LE, MacEwen GD. Treatment of congenital dislocation of the hip in children between the ages of one and three years. $J$ Bone Joint Surg Am. 1986;68:829-846. 